\title{
Utilización de zapatilla distal debido a pérdida prematura de una segunda molar temporal: Reporte de caso
}

\section{Use of Distal Shoe for Premature Loss of Second Temporal Molar: A Case Report}

\author{
Natalia Gutiérrez Marín DDS¹ \\ 1. Profesora, Instructora. Universidad de Costa Rica, Costa Rica. \\ Autor de Correspondiencia: Dra. Natalia Gutiérrez Marín - natalia.gutierrez@ucr.ac.cr \\ Recibido: 25-IV-2014 \\ Aceptado: 22-X-2014 \\ Publicado Online First: 15-XI-2015
}

D0I: http://dx.doi.org/10.15517/ijds.v0i0.21180

\section{RESUMEN}

El mantenimiento de la dentición temporal hasta su momento natural de recambio es muy importante para guiar la erupción de piezas permanentes y guardar el espacio de las mismas. Una pérdida prematura de piezas temporales puede producir: disminución del perímetro del arco, desviación de la línea media, maloclusiones, erupciones ectópicas, apiñamientos, impactación o extrusión de piezas permanentes, anomalías en la mucosa y adquisición de hábitos parafuncionales. Si se extrae tempranamente una segunda molar temporal, se puede utilizar una zapatilla distal como mantenedor, el cual posee una técnica complicada y sensible, además de que no está indicado para todo tipo de pacientes. Se reporta el caso de la colocación de una zapatilla distal en el cuadrante inferior derecho en una niña de 5 años de edad, se muestra la técnica empleada así como las ventajas y desventajas de la misma y el seguimiento que se dio durante un año.

\section{PALABRAS CLAVE}

Zapatilla distal, Intraalveolar, Mantenedor de espacio,

Pérdida prematura de piezas dentales.

GUTIÉRREZ, N., 2015: Utilización de zapatilla distal debido a pérdida prematura de una segunda molar temporal: Reporte de caso.-ODOVTOS-Int. J. Dental Sc., 17-1 (January-April): 21-29 . 


\section{ABSTRACT}

The maintenance of the temporal dentition until its natural replacement is very important, due to the fact that the primary pieces are in charge to secure the permanent pieces' space, in addition to guide the eruption process. The premature loss of temporal pieces could lead to: decrease in arch perimeter, desviation of the mid line, malocclusions, ectopic eruptions, crowding, impact and/or extrusion of permanent pieces, abnormalities in the mucous and acquisition of parafunctional habits. If early second molars extracted, can be used as a distal shoe maintainer; which is a complicated and sensitive technique, plus it is not suitable for all patients. This case report describes the use of a distal shoe in the inferior quadrant of a 5 years old child, the technique used, advantages and disadvantages of the appliance, also monitoring for one year.

\section{KEYWORDS}

Distal shoe, Intra-alveolar, Space maintainer, Premature tooth loss.

\section{INTRODUCCIÓN}

Uno de los factores más importantes con respecto a la odontología preventiva e interceptiva es la conservación de la dentición primaria hasta su momento natural de exfoliación, ya que los dientes temporales son los responsables de conservar el espacio para la alineación de los dientes permanentes (1).

A pesar de las medidas preventivas e interceptivas que se emplean en la actualidad, la realidad es que se continúa extrayendo piezas temporales tempranamente. Se debe tener en cuenta que los molares temporales albergan entre sus raíces los gérmenes de los premolares, por lo que la realización de tratamientos con pronósticos dudosos en molares temporales no está indicado, ya que sería un riesgo para el germen en desarrollo y, por lo tanto, en muchas ocasiones la extracción es el tratamiento a elegir (2).

Se considera pérdida prematura cuando los dientes temporales se exfolian o son extraídos antes del momento fisiológico de recambio, con menos de las tres cuartas partes o la mitad de la raíz del diente sucedáneo formado, 0 bien si existe más de $1 \mathrm{~mm}$ de hueso alveolar cubriendo el sucesivo diente permanente. (3-4).
La pérdida prematura puede producir: disminución del perímetro del arco, desviación de la línea media, maloclusiones, erupciones ectópicas, apiñamientos, impactación de piezas permanentes, anomalías en la mucosa, extrusión de piezas permanentes y adquisición de malos hábitos orales (5-8).

Los primeros estudios sobre la pérdida de espacio datan de 1965, donde se observó un significativo movimiento mesial de la primera molar permanente cuando se había extraído una segunda molar temporal y no se utilizó algún mantenedor de espacio (9).

La pérdida prematura de una segunda molar temporal inferior puede producir una disminución significativa en la longitud del perímetro del arco y una discrepancia alveolo dentaria, pues la primera molar permanente erupciona en una posición mesializada y lingualizada (10-11).

Varios estudios muestran que la pérdida de espacio es mayor en la mandíbula que en la maxila, y mayor si la pieza extraída es una segunda molar que una primera molar temporal (12). Se ha reportado que, aproximadamente, el $51 \%$ de la pérdida prematura de una primera molar temporal $y$ de un $70 \%$ de una segunda molar temporal 
resultará en una pérdida del perímetro del arco, con una consecuente malposición de las piezas permanentes (1).

Este espacio perdido tiende a convertirse en casos severos cuando coexiste con apiñamientos, sobremordidas, erupciones ectópicas 0 una desfavorable relación molar (13).

Existen factores que se deben tomar en cuenta a la hora de considerar el mantenimiento del espacio tras la pérdida prematura de piezas temporales (14).

- Tiempo transcurrido desde la pérdida.

- Edad del paciente: no tanto la cronológica, sino la de su desarrollo dentario.

- Cantidad de hueso que recubre al diente no erupcionado.

- Secuencia de erupción.

- Erupción retardada de dientes permanentes.

- Ausencias congénitas de piezas permanentes.

La mayor pérdida de espacio ocurre durante los primeros 6 meses, posterior a la extracción. Por esto, lo mejor es colocar un mantenedor de espacio tan pronto como sea posible (15).

Los mantenedores de espacio se utilizan para tratar de evitar las complicaciones citadas cuando se extraen prematuramente piezas temporales y pueden ser fijos o removibles $(9,16)$.

En el caso de la pérdida prematura de una segunda molar primaria, el mantenedor más ampliamente utilizado es la zapatilla distal (0 también Ilamado intraalveolar). En 1930, Willbett presentó el primer mantenedor con una extensión distal y lo llamó "distal shoe" (17).

La zapatilla distal está formada por una banda o corona de acero cromado colocada en la primera molar temporal, la cual tiene soldada un alambre que se une a una extensión distal subgingival que se localiza en la superficie mesial de la primera molar permanente sin erupcionar (1). Para que el intraalveolar sea efectivo, la extensión distal debe extenderse dentro del proceso alveolar en contacto con la superficie de la primera molar permanente, aproximadamente $1 \mathrm{~mm}$ por debajo del reborde mesial. Una vez que la molar haya erupcionado, la zapatilla distal debe reemplazarse por una banda y asa (9).

Existen intraalveolares que vienen prefabricados para colocarlos en una sola cita y no recurrir a los servicios de un laboratorio dental, algunas de las casas comerciales que los fabrican son: Denovo, Calif y Dental Supply Co. (18).

Para colocar una zapatilla distal, debe realizarse un control radiográfico para determinar el momento en que el germen del primer molar permanente comienza a estar en una posición extraósea $(2,19)$.

El diagnóstico del desarrollo de maloclusiones en la dentición primaria no contraindica la utilización de la zapatilla distal, inclusive si el paciente requiriera utilizar ortodoncia, el uso del intraalveolar estaría indicado, porque al evitar la mesialización de la primera molar permanente se facilita el tratamiento ortodóntico a futuro (1).

Un estudio realizado en monos, a los cuales se les colocaron extensiones intragingivales de acero inoxidable para valorar la respuesta de los tejidos blandos, obtuvo como resultado que, luego de 4 semanas, se observaba un epitelio bien organizado cubriendo el tejido conectivo adyacente a las extensiones; sin embargo, en algunos casos también se observó un estado crónico de una inflamación leve; por lo que los investigadores concluyeron que la zapatilla distal es una opción para pacientes con buen estado de salud, no así en infantes sistémicamente comprometidos (20). 
Algunos inconvenientes de este tipo de mantenedor de espacio son: en el aspecto biológico, mantienen una comunicación constante entre la cavidad bucal y el hueso, estableciendo un riesgo de que se produzca una infección crónica en la zona e incluso una osteomielitis; además, puede no cumplir su objetivo de guiar a la primera molar si el germen que presenta está desplazado en sentido vestibulolingual, ya que la parte intragingival podría no realizar su labor de "tope" (2).

El caso que se presenta adquiere impacto clínico desde el punto de vista docente, debido a que aproxima al estudiantado a emplear uno de los mantenedores de espacio menos usado en la Odontopediatría, no porque carezca de utilidad, pues guía la erupción de la primera molar permanente en caso de extracción prematura de una segunda molar temporal, sino porque muchos clínicos relacionan la colocación de la zapatilla distal con un procedimiento complejo y, por lo tanto, no lo usan con frecuencia.

\section{REPORTE DEL CASO}

A continuación se describe un caso donde se utilizó una zapatilla distal; la madre de la niña autorizó la publicación del mismo.

En la consulta privada de la Clínica María Cecilia, ubicada en la ciudad de Cartago, se presentó una niña de 5 años de edad, a la cual hace 4 meses, en otra clínica dental, le extrajeron, por caries, la segunda molar temporal inferior derecha. La queja principal de la madre fue: "necesito que le coloquen un aparato para que no se le muevan las muelas a mi hija".

Al realizar la anamnesis, la niña no presentó alguna condición sistémica de relevancia ni hábitos orales Respecto de su comportamiento, es muy bueno y colaborador.
La madre indicó que, luego de la extracción, está muy pendiente de la higiene bucal de la niña porque comprendió la magnitud de los problemas que puede acarrear un aseo deficiente. La niña se presenta con buena higiene oral, al hacer un recuento de biofilme dental, el promedio que se obtiene es de 0,7 .

En el examen clínico se observó el espacio correspondiente a la segunda molar temporal inferior derecha, las primeras molares temporales superiores obturadas y la primera molar temporal inferior derecha con sellante de fosas y fisuras.
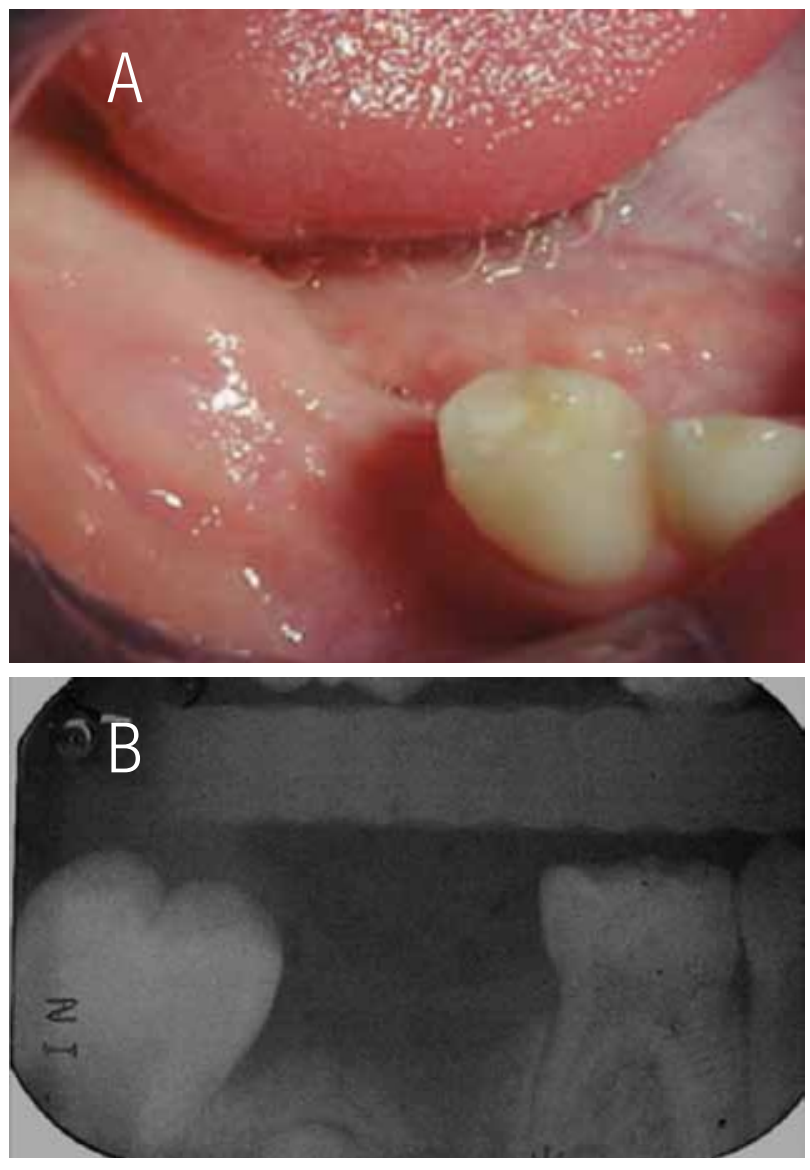

Figura 1. A. Zona de la segunda molar temporal extraída prematuramente. B. Radiografía del área de la molar extraída.

Posterior a la toma de una radiografía periapical, para verificar si la primera molar permanente estaba en una posición extraósea, se planeó realizar una zapatilla distal, por lo que 
en una segunda cita se procedió a adaptar una banda marca $3 \mathrm{M}$ en la primera molar temporal inferior derecha y se tomó una impresión con alginato marca Ortogelflex para confeccionar el aparato de forma indirecta, la impresión se vació con yeso amarillo marca Whip Mix. Se utilizó una banda y no una corona de acero cromado, dado que la primera molar temporal inferior derecha se encontraba sana y no era necesario realizar desgastes para colocar una corona. El modelo se envió al laboratorio dental para confeccionar el mantenedor. Para evitar interferencias en la oclusión, se realizó una ondulación en el asa del intraalveolar. Con la radiografía tomada, se estableció la distancia a la cual debía de ubicarse la extensión distal.

Una vez confeccionada la zapatilla distal, se citó a la paciente para su colocación. Se anestesió la zona retromolar inferior derecha con una técnica infiltrativa y anestésico de la marca Scandonest al $2 \%$. Se procedió a realizar una incisión en la que se introdujo la proyección distal del intraalveolar. Se colocó en boca, el mantenedor de espacio y se le hicieron varios ajustes. Se tomó una radiografía para el debido control, donde se observó la proyección en íntimo contacto con la superficie mesial de la primera molar permanente.
Se adherió el aparato con cemento de ionómero de vidrio marca 3M (Figura 2). No se prescribió antibiótico, ya que la incisión fue pequeña y no tiende a producir algún tipo de infección. Se recetó acetaminofén para tomar solo en caso de presentar dolor.
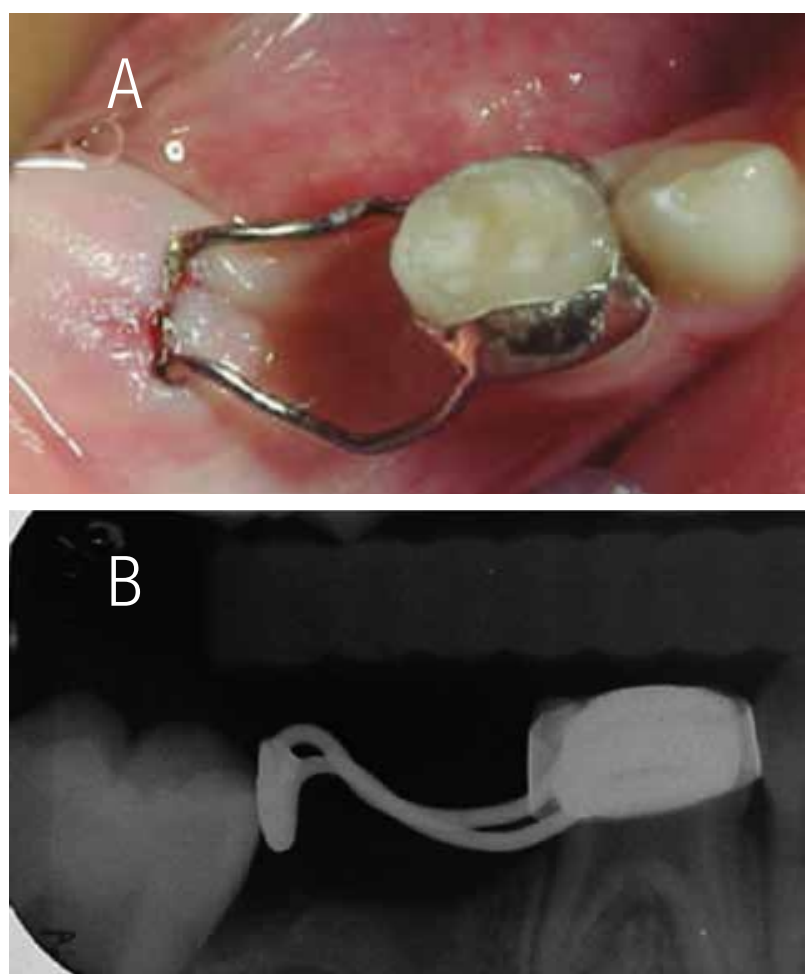

Figura 2. A. Zapatilla cementada en boca. B. radiografía con la extensión infragingival en contacto con la primera molar permanente. 
En la cita de control, a la semana siguiente, se observó que la incisión no estaba infectada, la zapatilla distal no ocasionaba problemas y la madre de la niña indicó que solo le suministró acetaminofén una vez, el día de la colocación, porque presentó un ligero dolor.

La niña se citó en varias ocasiones para valorar la higiene oral y la correcta colocación del intraalveolar.

A los 7 meses de colocada la zapatilla distal, se observó la erupción de la porción mesial de la primera molar permanente inferior derecha. A los 11 meses, ya se apreciaba la mayoría de la cara oclusal de la molar.
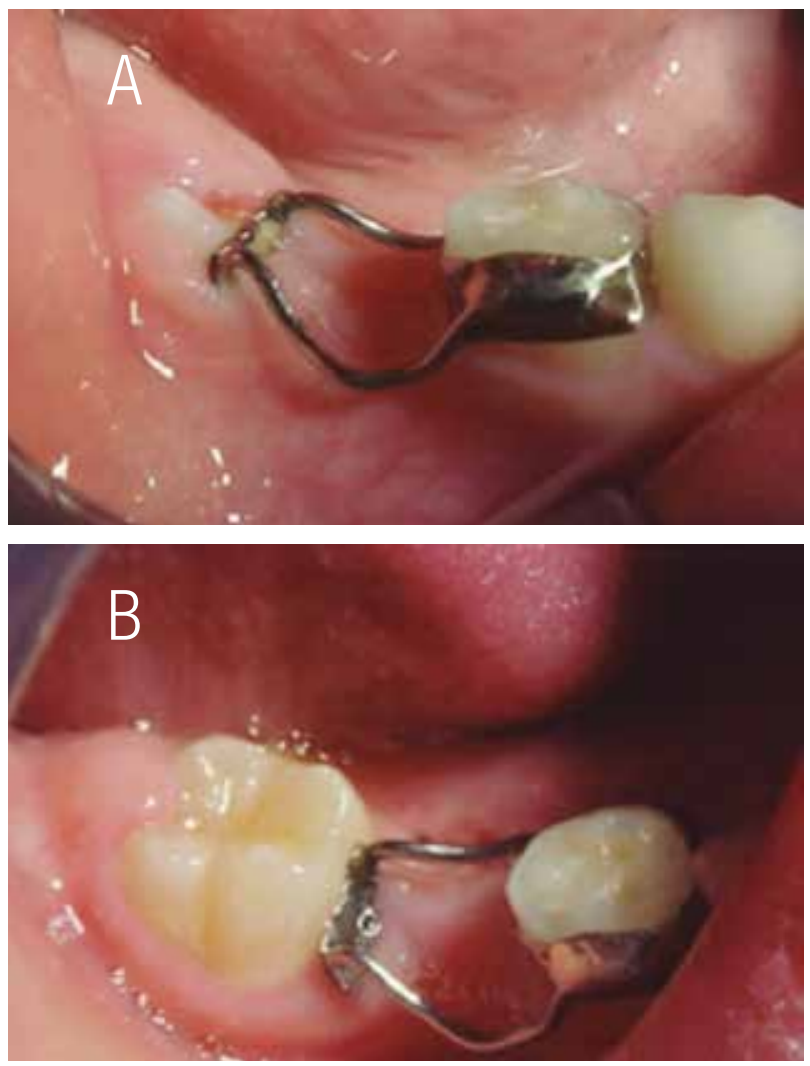

Figura 3. A. Molar permanente erupcionando a los 7 meses de colocada la zapatilla. B. Mayor parte de la molar permanente erupcionada.

Una vez erupcionada la molar permanente, se procedió a retirar el intraalveolar y sustituirlo por un banda y asa.
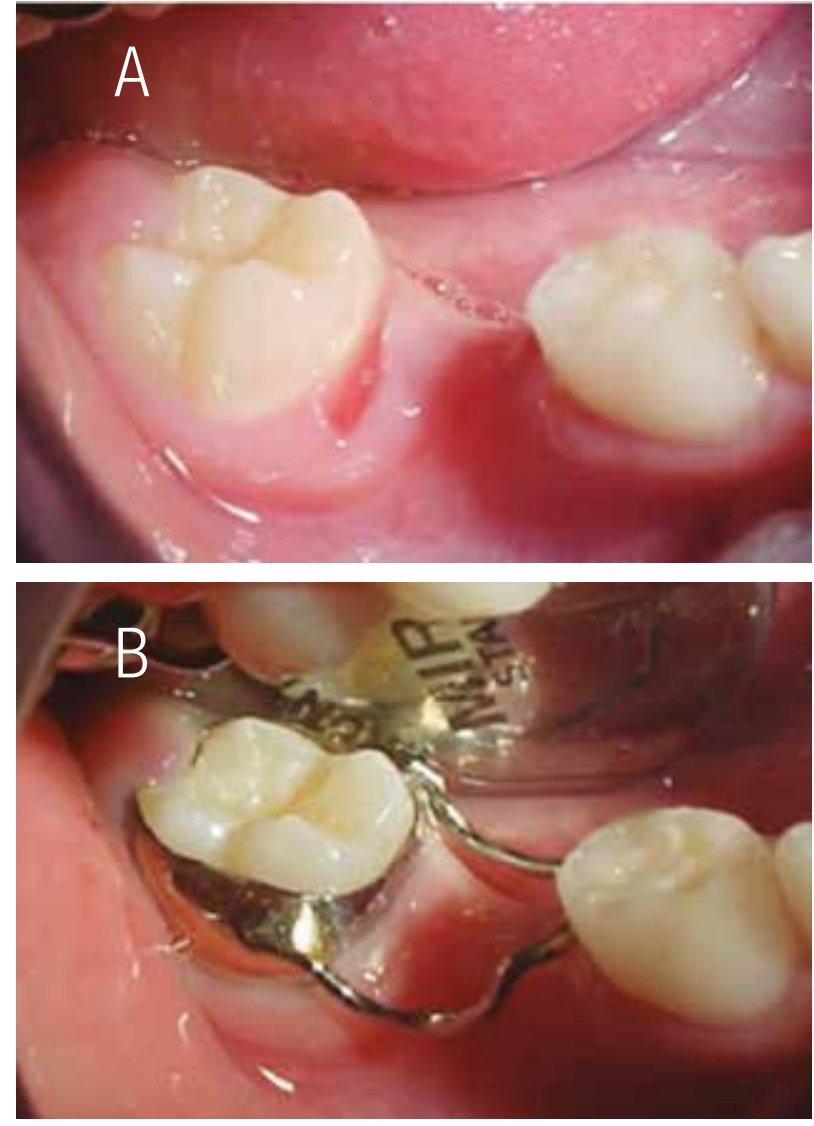

Figura 4. A. Retiro de intralveolar. B. Colocación de banda y asa.

\section{DISCUSIÓN}

Se decidió utilizar una zapatilla distal, ya que como cita Barbería et al. (17), la decisión sobre cuál mantenedor de espacio se debe utilizar se basa en factores generales y locales relacionados con el niño, la colaboración de sus padres y la experiencia del odontólogo en el uso de los diferentes tipos. En este caso, tanto la niña como la madre estuvieron en la mayor disposición de colaborar, y no se utilizó un aparato prefabricado, debido a que no se tenía experiencia en la técnica.

Por otra parte, la niña no presentó situaciones especiales que contraindicaran la colocación de la zapatilla distal como: pérdida prematura de múltiples piezas, deficiente higiene oral, condiciones sistémicas tales como discrasias sanguíneas, inmunosupresión, defectos cardiacos, 
fiebre reumática, diabetes 0 un estado generalizado de debilidad $(1,17,21)$.

A la hora que se tomó la radiografía, se observó que la primera molar permanente se encontraba en una posición extraósea, por lo tanto el momento ideal para colocar el intraalveolar hubiera sido el mismo día que se extrajo la molar temporal, situación que no se dio porque en la clínica donde le extrajeron la pieza no le ofrecieron la opción del mantenedor. En el momento de utilizar este mantenedor de espacio, no se presentó una de las mayores desventajas de la zapatilla distal: su técnica de colocación complicada y muy sensible. Lo anterior, dado que es difícil lograr un contacto pasivo de la extensión distal con la superficie de la molar aún sin erupcionar; si esta extensión no se coloca en el lugar correcto, puede provocar defectos en la segunda premolar en formación (11). Esta desventaja del intraalveolar se puede solventar, en gran parte, si se toman radiografías e impresiones de calidad y además, se trabaja con un técnico dental que posea experiencia en la confección de zapatillas distales.

La extensión distal del intraalveolar debe quedar en íntimo contacto con la superficie mesial de la primera molar permanente y a $1 \mathrm{~mm}$ por debajo del reborde mesial (9). En este caso, si se logró el íntimo contacto, no así que quedara a $1 \mathrm{~mm}$ por debajo del reborde; sin embargo, el mantenedor sí fue exitoso en guiar la erupción de la primera molar permanente.

La primera molar permanente tardó 11 meses en aparecer en la cavidad oral desde que se realizó la extracción de la segunda molar temporal y 7 meses desde que cementó la zapatilla distal. Como se aprecia en la radiografía de control, la molar permanente ya presentaba una pequeña inclinación mesial al momento de cementar el intraalveolar porque habían transcurrido 4 meses desde la extracción de la molar temporal. De no haber colocado el mantenedor, se podría haber producido una pérdida significativa de espacio, ya que según muestran cuantiosos autores $(1,11$, 22-23), una pieza en erupción presenta mayor potencial para producir pérdida de espacio que una pieza ya erupcionada.

Durante estos 7 meses que tardó la primera molar permanente en erupcionar, no se observó una extrusión de la pieza antagonista a la segunda molar temporal extraída, situación que pudo presentarse, ya que la zapatilla distal no repone la función masticatoria ni impide la sobrerupción de las piezas antagonistas. $(4,24)$.

Al retirar el intraalveolar se observó que la zona donde estaba la proyección distal mostraba un tejido levemente enrojecido sin muestras de sangrado, agrandamiento o infección, lo que concuerda con los resultados expuestos por Mayhew et al. (20) en su estudio sobre la respuesta tisular a objetos intragingivales.

La literatura muestra que el intraalveolar es uno de los mantenedores de espacio menos utilizado. En el ámbito de la práctica privada, un estudio realizado por Al-Dlaigan (25), en Arabia Saudita, mostró que de los 236 odontólogos entrevistados solo 2 de ellos colocaron un intraalveolar $(0,8 \%$ de todos los mantenedores que fueron empleados). En el área pública, en la Universidad de Costa Rica, de un total de 340, solo el 4,4\% fueron intraalveolares (26). Ambos estudios refieren que la técnica es compleja, lo que podría incidir en la poca utilización del mismo, pero como se puede observar en este caso, si se siguen los pasos establecidos, el empleo de la zapatilla distal no resulta tan complicado, por lo que podría enseñarse más ampliamente la técnica a los y las estudiantes de odontología para que en un futuro la puedan implementar en sus prácticas privadas. 


\section{CONCLUSIONES}

La utilización de la zapatilla distal fue exitosa, evitando la pérdida de espacio producto de la extracción temprana de la segunda molar temporal inferior, debido a que la primera molar permanente erupcionó en buena posición guiada por la extensión distal del intraalveolar. Por lo anterior, la zapatilla distal es un mantenedor de espacio con una técnica un poco compleja, pero si se utiliza en el momento adecuado puede evitar complicaciones en el futuro.

\section{REFERENCIAS}

1. Dhull K, Bhojraj N, Yadav S, Prabhakaran S. Modified distal shoe appliance for the loss of a primary second molar: a case report. Quintess Int 2011;42(10):829-833.

2. Barbería E, Cuesta L, Lara A, Maroto M. Problemas clínicos en pérdida prematura de los segundos molares temporales. El mantenedor de extremo libre, como una alternativa. Gaceta Dental 2010. 215, 102-115.

3. García Y, Da Silva de Carballo L, Medina C, Crespo O. Efecto de la pérdida prematura de molares primarios sobre la relación horizontal incisiva. Revista de Odontopediatría Latinoamericana 2011;1(1):49-57.

4. Ram D, Ben-Israel M, Efrat J, Moskovits M. Mantenedores de espacio mandibulares para la prevención de pérdida de espacio, posterior a la pérdida prematura de molares primarios. Odontol Pediatr 2010;9(2):127-140.

5. Moore T, Kennedy D. Bilateral Space Maintainers: A 7-year Retrospective Study from Private Practice. Pediatr Dent 2006;28: 499-505.

6. Moreno S, Pedraza G, Lara E. Mantenedor de espacio en pérdida prematura de órganos dentarios en dentición mixta: Reporte de un caso. Revista ADM 2011; 68(1):30-34.

7. Sasa I, Hasan A, Qudeimat M. Longevity of Band and Loop Space Maintainers Using Glass Ionomer Cement: A Prospective Study.
European Archives of Pediatr Dent 2009;10 (1):6-10.

8. Simsek S, Yilmaz Y, Gurbuz T. Clinical evaluation of simple fixed space maintainers bonded with flow composite resin. J Dent Child 2004;71(2):163-168.

9. Laing E, Ashley P, Farhad BN, Daljit SG. Space maintenance. Int $\mathrm{J}$ Paediatr Dent 2009;19:155-162.

10. Cameron A, Widmer R. Manual de Odontología Pediátrica. Madrid: Harcourt; 1998.

11. Kirshenblatt S, Kulkarni G. Complications of surgical extraction of ankylosed primary teeth and distal shoe space maintainers. J Dent Child 2011;78(1):57-61.

12. Tunison W, Flores C, ElBadrawy H, Nassar U, El-Bialy T. Dental arch space changes following premature loss of primary first molars: a systematic review. Pediatr Dent 2008; 30: 297-302.

13. Nouri M, Kennedy D. Optimal fit of chairsidefabricated distal shoe space maintainer. Eur Arch of Paediatr Dent 2013;14(1):351-354.

14. Esaú A, Ribelles W, Sáenz S, Bellet L. Zapatilla distal como tratamiento en pérdida prematura de un segundo molar primario. Revista de Operatoria Dental y Endodoncia 2006; 5(39).

15. Baroni C, Franchini A, Rimondini L. Survival of different types of space maintainers. Pediatr Dent 1994;16(5):360-361.

16. McDonald R, Avery D. Odontología Pediátrica y del adolescente. $6^{\mathrm{a}}$ ed. España: Harcourt Brace; 1998.

17. Barbería E, Lucavechi T, Cárdenas D, Maroto M. Free-end space maintainers: desing, utlization and advantages. J Clin Pediatr Dent 2006; 31(1), 5-8.

18. Brill Warren. The distal shoe maintainer: chairside fabrication and clinical performance. Pediatr Dent 2002; 24(6):561-565.

19. Diab Mai. Primary failure of eruption of primary molars: a review and case report. Quintess Int 2001; 32(1):55-60. 
20. Mayhew M, Dilley G, Dilley D, Jacoway J, Johnson P. Tissue response to intragingival appliances in monkeys. Pediatr Dent 1984;6(3):148-152.

21. Gujjar K, Indushekar K, Amith H, Sharma S. Modified distal shoe appliance-fabrication and clinical performance. J Dent Child 2012; 79(3):185-188.

22. Gegenheimer R, Donly K. Distal shoe: a cost effective maintainer for primary second molars. Pediatr Dent 1992; 14(4):268-269.

23. Subekti A, Kuswandari S. The use of Crown (SSC) and Loop as Space Maintainer in Premature Loss of Mandibular Second
Primary Molar on Children Aged 5 years. (Case Report). Indonesian J Dent Res 2013; 5(1):188-191.

24. Pinkham J, Cassamassino P, Fulds H, Mc Tique D, Nowak A. Odontología Pediátrica. México: Editorial McGraw; 1996.

25. Al-Dlaigan Yousef. A survey of the use of space maintainers by private dentists in Riyadh-Saudi Arabia. Pakistan Oral Dent J 2008. 27(1):39-44.

26. Gutiérrez N, López A. Mantenedores de espacio colocados del 2008 al 2011 en la Facultad de Odontología, Universidad de Costa Rica. Odovtos 2013; 15:13-19. 